\title{
Mechanism Simulation and Experiment of 3-DOF Parallel Robot Based on MATLAB
}

\author{
$\mathrm{Ma} \mathrm{Li}^{1,}$ a , Dexue $\mathrm{Bi}^{2, \mathrm{~b}}$ and Zhipeng Xiao ${ }^{3, \mathrm{c}}$ \\ ${ }^{1}$ School of Mechanical Engineering, Tianjin University of Science and Technology, Tianjin, China \\ ${ }^{2}$ School of Mechanical Engineering, Tianjin University of Science and Technology, Tianjin, China \\ ${ }^{3}$ School of Mechanical Engineering, Tianjin University of Science and Technology, Tianjin, China

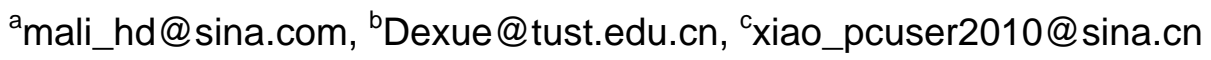

Keywords: DELTA Parallel robot, 3D modeling, MATLAB simulation, Experiment

\begin{abstract}
This paper designed a kind of DELTA parallel robot to realize the seed sorting, which simplifies the FANUC(M-1iA) parallel robot in instance structure. SolidWorks is used to establish a 3d model and converts it into a SimMechanics model. After that simple movement simulation experiments is carried out to verify the correctness of the model. Five order polynomial curves are used in planning portal grabbing path. Based on the established simulation model, PID control algorithm is designed and tested, whose results can meet the precision of the design requirements. Based on the proceeding investigation, a seed-sorting comprehensive simulation experiment is designed; the seed-sorting and locating is completed and simulation results are verified. The robot hand for grasp and absorb is installed on FANUC (M-1iA) parallel robots. Sorting and locating experiment results is simulant to that of the simulation.
\end{abstract}

\section{Introduction}

Compared with the traditional serial robots, parallel robot has prominent features such as high rigidity, high bearing capacity, high precision, small error, low motion inertia and inverse kinematic solutions easily, which makes parallel robot widely used in light industrial automation production line such as food, medicine, electronic, etc. DELTA robot is a kind of parallel mechanism, which had been put forward in 1985. It is famous for triangular shape of the base platform and the motion platform. DELTA robot can realize quickly grabbing and placing the light small objects and usually be used in the assembly of small components operations [1,2,7]. In this paper, we designed a 3-DOF parallel robot that simplifies the FANUC and carried on the analysis, the simulation and experiment.

\section{Imitation of the DELTA Robot Structure Design and Kinematics Analysis}

\section{A. Parallel Robot Structure}

In this article, instantiation design seed sorting robots consults the structure of FANUC (M - liA) high speed parallel robot to design and on this basis to simplify. 3d entity model is set up under the SolidWorks (Fig.1).

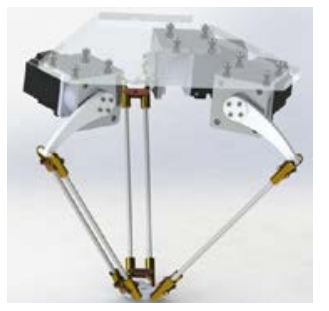

Fig.1 3d Model assembly drawing

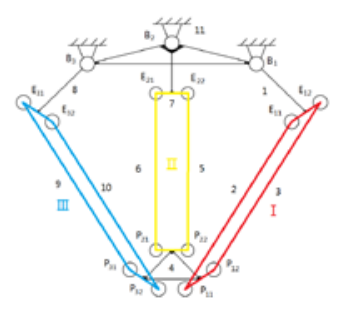

Fig.2 Parallel robot mechanism diagram

Parallel four-bar linkage and active arm geometrical structure ensure that moving platform and static platform remain parallel. The robot end actuator can only move along the $\mathrm{X}-\mathrm{Y}-\mathrm{Z}$ axis in 
rectangular coordinate (Fig.2) [3].

\section{B. Kinematics Analysis}

Since the structure of DELTA parallel robot is center symmetry, a separate analysis of the branched chain can be applied to the other two branched chain. Rectangular coordinate system has been established (Fig.3). The static platform geometric center is considered as the origin $\mathrm{O}$ to establish rectangular coordinate system $\mathrm{X}-\mathrm{Y}-\mathrm{Z}$. A static platform radius is $\mathrm{R}$; moving platform radius is $\mathrm{r}$; the active arm length is $l_{1}$; the slave arm length is $l_{2}$; the three angles of the three active arms and $\mathrm{x}-\mathrm{y}$ plane are $\theta_{1}, \theta_{2}$ and $\theta_{3}$; the three angles of the three active arms with the $\mathrm{X}$ axis are $\alpha_{1}, \alpha_{2}$ and $\alpha_{3}[4]$.

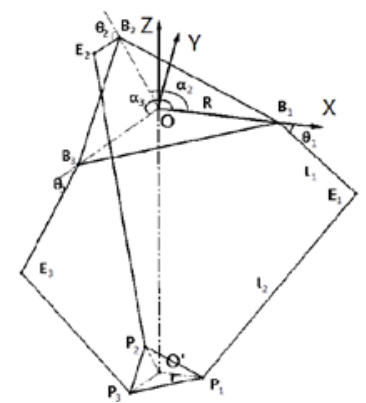

Fig.3 Rectangular coordinate system

$$
\theta_{i}=2 \arctan \left(\frac{-J_{i}-\sqrt{J_{i}^{2}+I_{i}^{2}-K_{i}^{2}}}{K_{i}+I_{i}}\right)
$$

Among them $(\mathrm{i}=1,2,3)$ :

$$
\begin{aligned}
& I_{i}=2 l_{1}\left(\left(x_{0}+r \cos \alpha_{i}-R \cos \alpha_{i}\right) \cos \alpha_{i}+\left(y_{0}+r \sin \alpha_{i}-R \sin \alpha_{i}\right) \sin \alpha_{i}\right) \\
& J_{i}=2 l_{1} z_{0} \\
& K_{i}=\left(x_{0}+r \cos \alpha_{i}-R \cos \alpha_{i}\right)^{2}+\left(y_{0}+r \sin \alpha_{i}-R \sin \alpha_{i}\right)^{2}+z_{0}^{2}+l_{1}^{2}-l_{2}^{2}
\end{aligned}
$$

\section{Select Institutions Size}

Consulting the structure size of FANUC (M - liA) robot, the appearance size of the robot is achieved by measuring. The measurements are taken as the basic data, and a parameter is chosen as a variable and others are fixed. In consideration of the working environment, the size of robot size should be as small as possible [5]. The appearance size of robot that is chosen is shown in the following Table1:

Table1. Installation Dimensions of Robot

\begin{tabular}{lllllll}
\hline $\mathrm{R}$ & $\mathrm{r}$ & $\mathrm{l}_{1}$ & $\mathrm{l}_{2}$ & $\alpha_{1}$ & $\alpha_{2}$ & $\alpha_{3}$ \\
\hline 100 & 40 & 100 & 270 & 0 & $120^{0}$ & $-120^{0}$ \\
\hline $\mathrm{X}_{\min }$ & $\mathrm{X}_{\max }$ & $\mathrm{y}_{\min }$ & $\mathrm{y}_{\max }$ & $\mathrm{h}_{\min }$ & $\mathrm{h}_{\max }$ & $\theta$ \\
\hline-230 & 194 & -218 & 218 & -365 & -217 & {$\left[0,120^{0}\right]$} \\
\hline
\end{tabular}

\section{The Analysis of the Working Space}

The analysis of the working space is an importance in robot design. The robot's working space is the work scope of robotic end, which is an important index to measure the performance of robot [6]. Searching algorithm [7] is used to describe the robot working space in MATLAB (Fig.4):

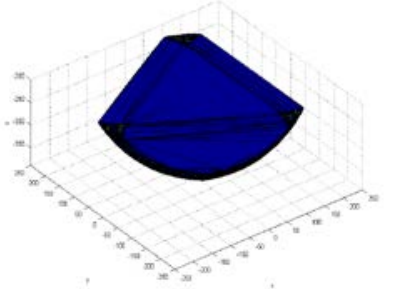

a. The robot working space

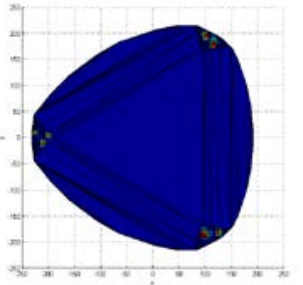

b. The robot working space $\mathrm{X}$ - Y plane 


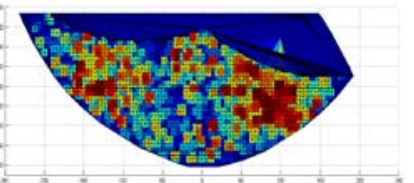

c. The robot working space $\mathrm{X}$ - Z plane

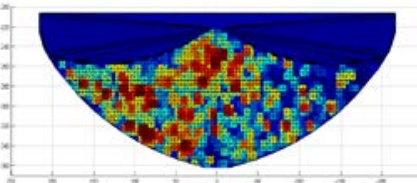

d. The robot working space Y-Z plane Fig.4

The size of working space $x \in[-232,192] ; y \in[-220,220] ; z \in[-362,-202]$ under 4 mm precision is approach the reference value, except maximum height. The maximum height $h_{\max }$ is calculated on the working space center. However, the maximum of working space may not in the center. As it is shown in the figure, the edge height of the workspace exceeds the center height, so there is error.

\section{The Establishment of the Kinematics Model Based on MATLAB}

The robot's physical properties are sorted according to the design of the seed. The physical model is established by using SimMechanics sublibrary (Fig.5).

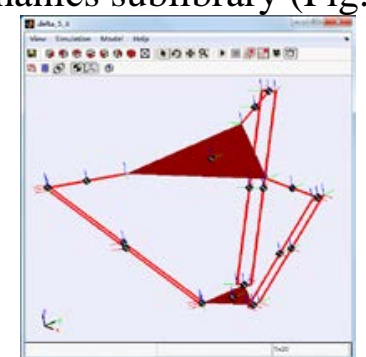

Fig.5 The physical model of the robot in MATLAB

This model is mainly composed of rigid body and joint module series, added the sensor in the joint. According to the design of the rigid body geometry, determine the relative position of each endpoint. For the model added physical parameters of the rigid body. Choose the same degrees of freedom of joints according to the actual structure joint module and set up the direction of joint axis. A single branched chain model:

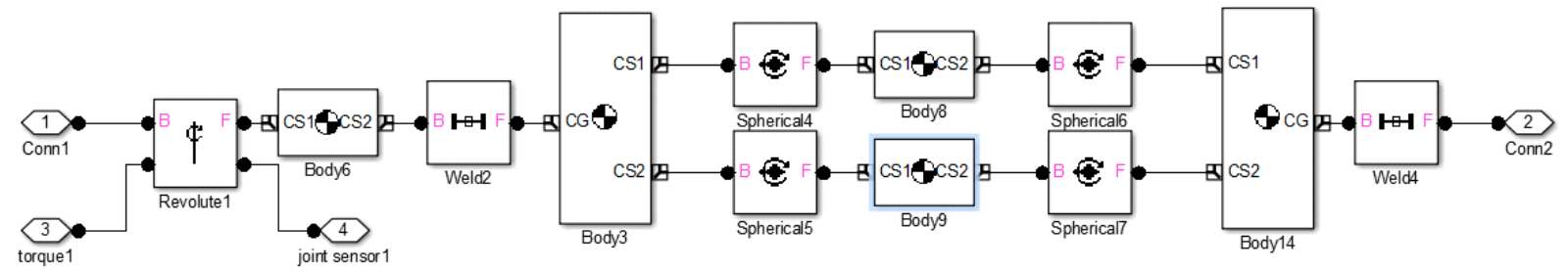

The overall physical model:

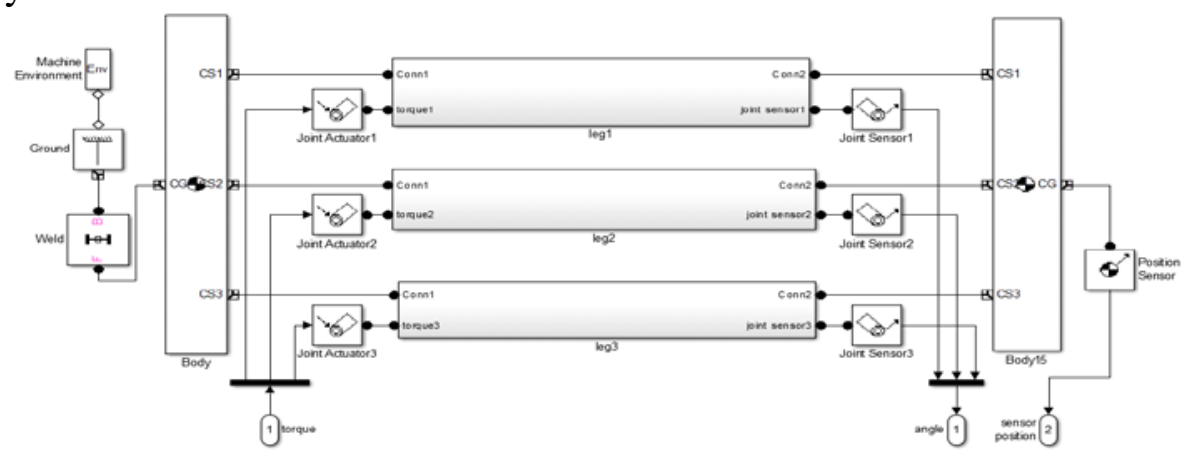

\section{Trajectory Planning}

The seed sorting robot designed in this paper mainly realizes drop operation, that is catching an object at A and dropping it at B. Avoidance path is used to avoid the collisions with other objects in the process of movement [8]. Generally, portal trajectory is applied (Fig.6). 


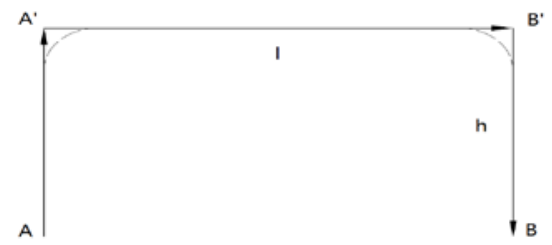

Fig.6 Portal trajectory

There are many kinds of trajectory function curves [9]. In this paper, under the condition of distance of movement $S_{\max }=250 \mathrm{~mm}$ and the total time $T=0.3 \mathrm{~s}$, five order polynomial curve and sine curve are compared in working space (Fig.7).

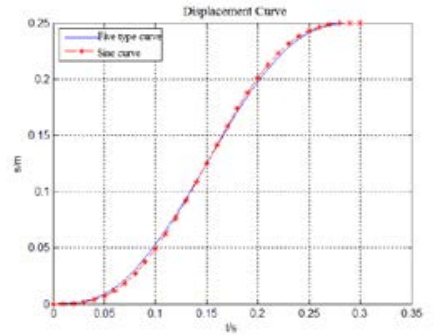

(1) Displacement curve

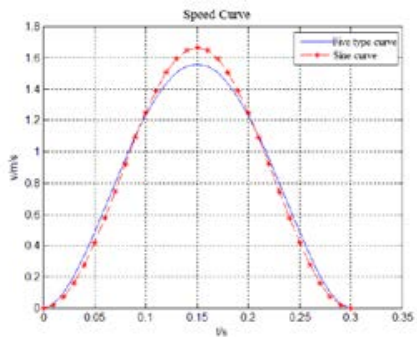

(2) Speed curve

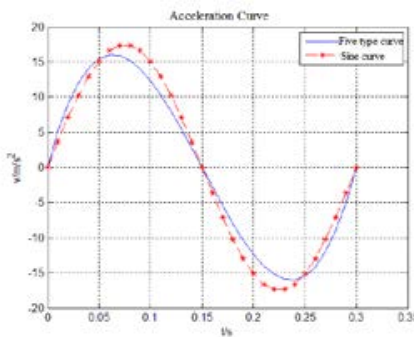

(3) Acceleration curve

Fig.7 Five order polynomial curve compared with sine curve

From the above curve comparison, we can see that when displacement curve is basically the same, the speed and accelerated speed's peaking data of sine curve is more than that of five order polynomial curve. Namely, the sine curve requires more than five order polynomial curve on the strength and driving force of the robot. So the movement characteristics of five order polynomial curve is better than sine curve in the end of perform.

\section{The Control Algorithm Research Based on Simulation Model}

This control system is a semi-closed loop control system to detect the feedback signals of the drive motor. Figure 8 is the robot control system block diagram:

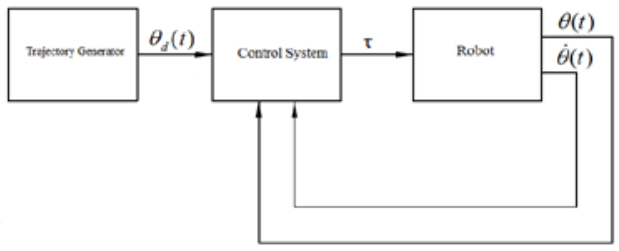

Fig.8 System control block diagram

PID control is a mature and widely used control method in control engineering. After a long period of engineering practice, it has formed a complete set of control method and the typical structure.

Through the method of trial and error, we determine that the scale coefficient ${ }^{K}{ }_{p}$ is 0.5 ; integral coefficient ${ }^{T}$ is 15; differential coefficient ${ }^{T}$ is 0.01 .

Overall control model like the following diagram:

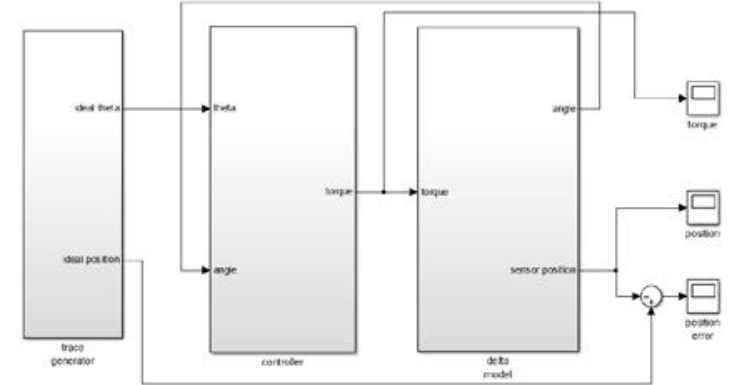

Based on the above overall control MATLAB Simulink model, the method same to simulation 
and model is adopted to control the robot port to perform the simplest R60 circular trajectory on Y-Z plane [10].

Running track through the sensor end (Fig.9):

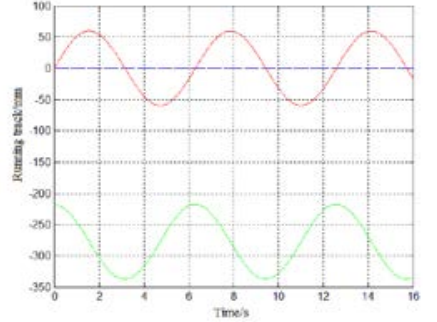

Fig.9 The running track

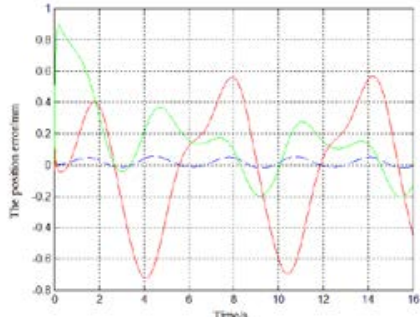

Fig.10 The position error

The running track is very close to theoretical path, which can conform to design purpose.

Spatial location error is beginning to have larger peak in the vertical direction because at the beginning of the moment there is no driving force. Under the action of gravity, the robot paw begins to move downward and have a larger error (Fig.10). But the control model can adjust it within the precision of $\pm 1 \mathrm{~mm}$ and control the error range, which meets the requirements of system control.

\section{Integrated Simulation Instance}

The image of seed on the belt is collected through video camera (Fig.11). Converting the image of seeds (Fig.12) into a gray image (Fig.13) can simplify the computer process and reduce the storage space.

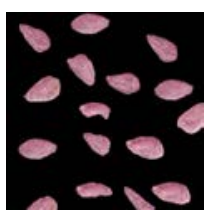

Fig.11 Original image

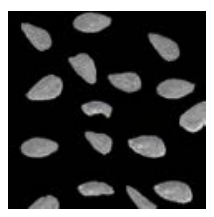

Fig.12 Gray image

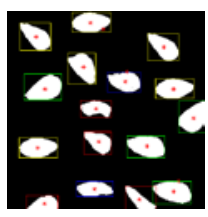

Fig.13 Results image

According to the size of the seed pixel area can distinguish different levels of seeds (Fig.13).

The robot sorting simulation can be realized through integrating simulation model and control algorithm.

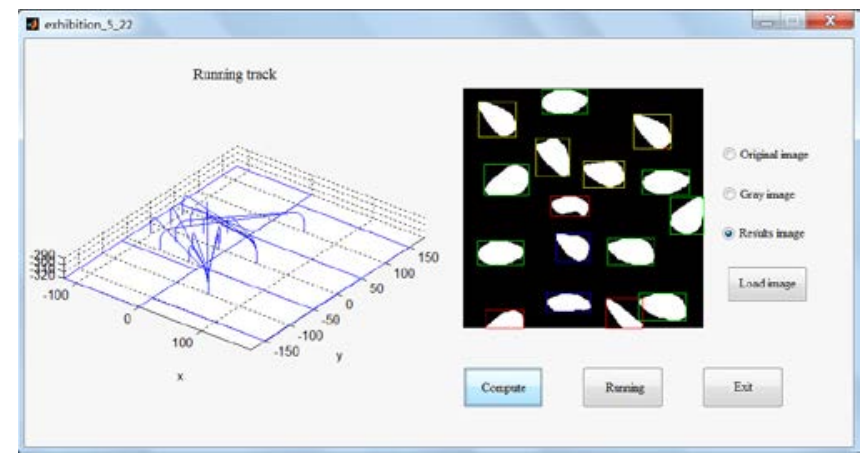

Fig.14 MATLAB user interface

The user's interface is designed by MTALB GUI function. The input image can automatically control the robot to sort out. The right side of Fig.14 is the processing area of loading image, which can observe the original image, gray image and detecting result. The left side is the calculated running track of seed sorting.

\section{Comprehensive Experiment}

Experiments of sorting coins of one Jiao, five Jiao and one Yuan are designed by using the FANUC (M - 1ia). The image is obtained and the information of abstaining coins' location is processed through video camera. The moving trajectory of robot paw is planned. The images are processed through MTALB and the obtaining of information of input image can automatically control the robot to complete the operation of sorting. Program is written and the coordinate 
position is inputted on FANUC (M - 1ia) parallel robot teaching and the moving trajectory is generated to realize the sorting of the coin. The execution process is shown below.

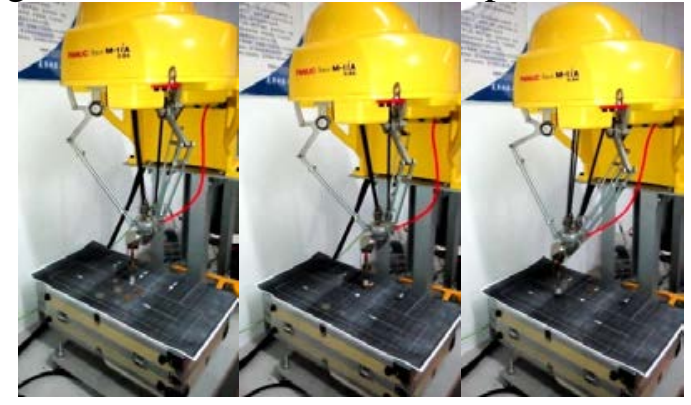

Fig.15 Coin sorting process

In the process, the paw of FANUC (M-1ia) parallel robot can complete that process of pick-and-place accurately, which shows that types of coins and the location information identified from the image can meet the requirements of sorting coins (Fig.15).

\section{Conclusion}

In this paper, by modeling the structure of FANUC (M-1ia), a three degree of freedom seed sorting parallel robot is designed. The inverse kinematics of the robot is calculated by researching kinematics. MATLAB is used as a research tool and the robot's working space is calculated and shown in MATLAB by using search method to write program, according to the results of the inverse kinematics reverse. The seed sorting physical model of parallel robot is established by using SimMechanics model in Simulink toolbox of MATLAB and simple motion and dynamic simulation experiment is carried out.

\section{References}

[1] Weishi Hua: Stacking robot based on DELTA institutions. Journal of Southwest Jiaotong University, Vol. 34(3) (1999), p. 290-294

[2] Guanglan Xia, Xiaoping Hu: The development status and prospect of parallel robot. China science and technology information, Vol. 22(2005), p. 28

[3] Zhao Jie, Yanhe Zhao, Hegao Cai. Geometric solution for direct kinematics of DELTA parallel robot[J]. Journal of Harbin Institute of Technology, Vol.35 (1) (2003), p. 25-27

[4] (America) John J.Craig: Introduction to Robotics [M].Beijing: China Machine Press (2012)

[5] Linggui Pu, Minggang Ji: Machine design [M]. Beijing: Higher Education Press (2004.5)

[6] Miller K: Maximization of workspace volume of 3-DOF spatial parallel manipulators [J]. Journal of Mechanical Design, Vol.124 (2002), p. 347-350

[7] Tian Tao: Design and Implementation of a High-Speed Pick and Place Parallel Robot [D]. Dalian University of Technology (2013)

[8] Qiming Tao: The Research of Robot's Trajectory Planning [D]. Hefei: Hefei University of Technology (2003)

[9] Huang Zhen, Lingfu Kong: Theory and control of parallel robot mechanism [M]. Beijing: China Machine Press (1997)

[10] Jinkun Liu: Design and simulation of robot control system on MATLAB. Tsinghua University Press (2008) 